\title{
Strip Theory for Underwater Vehicles in Water of Finite Depth
}

\author{
Jerome H. Milgram \\ Department of Mechanical Engineering \\ Massachusetts Institute of Technology
}

\begin{abstract}
This paper addresses the need to know the unsteady forces and moments on an underwater vehicle in finite depth water, at small enough submergences for it to be influenced by sea waves. The forces are those due to the waves themselves as well as the radiation forces due to unsteady vehicle motions. Knowledge of these forces and the mass distribution of the vehicle allow solution of the equations of motion at a single frequency. Since the theory is linear, any incident wave field can be decomposed into the sum of many individual single frequency sinusoidal waves. The motions due to each frequency component can then be added together to obtain the total predicted vehicle motions. The wave forces are due to the undisturbed sea wave plus those due to the diffracted wave necessary to satisfy boundary conditions on the vehicle. The long used strip theory for ships, with the inviscid flow approximation, is modified for finite depth and inclusion of lift forces on the vehicle fins. The two dimensional solutions for the forces on each strip are found by a different method than is commonly used for strip theory. This form of the theory is easier to deal with and requires much less computing time than a fully three-dimensional approach. Experiments are conducted and their results are compared with the theory. Excellent agreement is found between the theoretical and experimental wave forces, including the diffracted wave. It is shown that inclusion of the forces on the fins not only improves the theoretical wave forces, but also brings the results of theory for the radiation forces and moments due to vehicle motions much closer to the experimental values that the theory without inclusion of fin lift forces.
\end{abstract}

\section{Introduction}

During the past two decades, considerable development and use of autonomous underwater vehicles (AUV's) have taken place. They usually carry sensors for making one or more types of oceanographic measurements, ranging from chemical and biological sampling to bathymetric mapping, and more. A national defense application is detection of underwater, and even buried, mines by sonars on the vehicle. Measured data is either stored aboard or transmitted to a remote receiver by acoustic communications.

When an underwater vehicle operates near to the shore, it is usually in shallow water where it undergoes unsteady motions due to the unsteady forces and moments that sea waves generate on the vehicle. These motions interfere with the focusing of both acoustical and optical instrumentation. Motions in the vertical plane can lead to the vehicle hitting the bottom or broaching the free surface. Some positively buoyant vehicles can be difficult to re-submerge after broaching the surface. Motions in the horizontal plane interfere with course keeping and are troublesome when a vehicle is maneuvering into an underwater dock. Underwater docking is frequently used for data exchange and for vehicle battery charging.

Usually, wave sizes and water shallowness for safe vehicle operation are empirically determined. This can be a complex process since the deeper the water, the greater the tolerable wave height.

It would be helpful to be able to predict or estimate the unsteady fluid forces on a vehicle, due to waves and to unsteady vehicle motions. Together, the predictions can be used to calculate the unsteady vehicle motions. The term given to the analogous forces and motions of ships is "seakeeping", and this term is used here as well. Prediction or estimation of seakeeping forces not only allows motion computation for developing more rational limits of combinations of wave conditions and water shallowness for safe operation, but also can be used for optimizing vehicle control systems for shallow water operation. 
It should be noted that the problem of force and motion prediction for AUV's is fundamentally the same as for manned submarines. Only the scale is different since most AUV's are smaller than submarines.

Streamlined vehicles with a generally slender form will be considered, and for these the hydrodynamic methods used for the seakeeping of surface ships, with a few adjustments, are applicable. The unsteady motions which influence the track of the vehicle will be considered and these are: sway, heave, yaw and pitch. It will be assumed that the coupling of these motions with surge and roll are negligible. The near vertical plane symmetry of most AUV's makes roll moments and their couplings with other modes very small. For the special case of a vehicle with substantial deviations from vertical plane symmetry, sway-roll coupling would have to be added to the theory and solution method.

There is a wave induced roll moment, even on vehicles with absolute vertical plane symmetry due to the depth-dependent fluid motion in waves, but the roll moment is very small in comparison to other moments. However, the methods used here are entirely applicable to roll moments and motions if necessary. Because of the slenderness of the vehicle, unsteady surge forces are small in comparison to other forces. However, although the vehicles under consideration are globally slender, they are not locally slender near a blunt nose. Therefore, if one were to calculate the small surge forces by the Strip Theory presented here, the results of the calculation would, almost certainly, be erroneous.

Since a number of approximations will be made in the theory presented here, experimental validation is necessary to provide confidence in its use. The author and his students have conducted the needed laboratory experiments and some of their results will be provided. The first approximation that will be made in the theory is neglecting effects of fluid viscosity. Both an order of magnitude analysis and the large number of comparisons between experiments and inviscid theories for ship motions justify this approximation. A comprehensive discussion of the inviscid Strip Theory for ship motions and validating experimental results made by several investigators, for sway, heave, pitch, and yaw motions, is provided by Beck et al. [1]. This reference also contains a detailed bibliography of the major papers on strip theory. Since viscosity is neglected, a potential flow formulation is suggested even though it necessarily neglects the effects of vorticity in breaking waves.

Strip Theory for the seakeeping of surface ships has been developed over the past 50 years and used with great success. It is a quasi two-dimensional theory in which all the potential flow calculations are done in two dimensions with final results including the three-dimensional influence of forward speed. The theory is linearized in the incident wave amplitude and in the amplitudes of vehicle motion. This allows the hydrodynamic computations to be done for sinusoidal waves with simulations and statistics for random waves being done by means of a sum of sinusoids using the methods pioneered by St. Denis and Pierson [2]. An example of using this method for very long simulations, in a different problem, with high speed computation provided by use of Fast Inverse Fourier Transforms can be found in Milgram [3].

In recent years, fully three-dimensional computational methods for seakeeping have been developed, as exemplified by the computer program SWAN, written under the direction of Sclavounos [4]. However, these fully three-dimensional programs are quite demanding of computer resources and require a detailed gridding of both the vessel and the free surface. Therefore, Strip Theory has been chosen for use here. A number of specific differences exist between conventional strip theory of surface ships and its application to submerged vehicles in finite depth water which will be explained in the following sequel.

\section{Background}

Strip Theory of ship motions, as it is normally used, is a mathematically inconsistent approximation in the sense that some terms of various orders in beam/length are retained and others are absent. The theory applies to slender vehicles such that dimensions of all immersed cross sections are small in comparison to the vehicle length. Some terms in the free surface boundary condition are ignored, which are smaller than retained terms only for waves that are short in comparison to a subsequently explained limit based on the vehicle length, but which are impossible to evaluate within the context of the theory. The principal justification for use of strip theory is that it gives estimates for seakeeping motions and structural loads in ships with satisfactory engineering accuracy. Likewise, it is the comparison between theory and 
experiment that justifies use of strip theory for underwater vehicles. It is of interest that Ogilvie and Tuck [5] investigated Strip Theory for surface ships including all terms of leading order in beam/length and found that the normally omitted terms are small in comparison to what is retained. Descriptions of the background of strip theory are provided by Newman [6] (Chapter 7) and in the paper: "Ship Motions and Sea Loads" by Salvesen, Tuck and Faltinsen [7], who also developed a thorough formulation of the theory, hereinafter referred to as STF. For the past 35 years, STF and developments based on it have been the most widely used strip theories.

The theory provided here for underwater vehicles is a modified form of STF. The modifications used are:

1. The vehicle is submerged,

2. The water depth is finite,

3. Two-dimensional forces on cross sections are computed by using Green's Theorem,

4. The vehicle is presumed to end in a section having zero crossectional area so there are no transom corrections,

5. The hydrostatic restoring coefficients are related only to the vertical distance between the center of gravity and the center of buoyancy, with no free surface hydrostatic effects,

6. The forces and moments due to hydrodynamic lift forces on the vehicle fins are included.

7. The fore-and-aft (x) origin of the coordinate system is not taken in the $(y, z)$ plane of the center of gravity, and is chosen at midship for the calculations. This alters the terms in the "mass matrix" used in the equation of motion giving it the form used in [1].

The sixth item will be found to be important for four reasons:

a. The fin lift forces in comparison to the remaining forces are larger for a typical underwater vehicle than for a ship because the relative sizes of the underwater vehicle fins are larger,

b. For a submerged vehicle, the damping forces due to radiated waves are comparatively smaller than for surface ships.

c. Due to items (a) and (b) above, the damping of underwater vehicle motions is frequently dominated by fin lift forces and moments.

d. Since the hydrostatic restoring forces are small and the lift forces on the fins at non-zero angles of attack can be considerable, several terms in the restoring force matrix due to pitch and yaw motions are either augmented or entirely due to fin lift forces.

The same notation for the sectional added mass, damping coefficients and wave forces, as well as whole vehicle (spring-like) restoring coefficients, added mass, damping coefficients and wave forces and moments as used in STF are used here. Indices 1, 2, 3, 4, 5 and 6 refer to surge, sway, heave, roll, pitch and yaw respectively, although surge and roll and their influences are neglected here on the presumptions that the vehicle is slender and roll is unimportant for the problems to be solved. The potential flow (ignoring fin lift forces) sectional added masses and damping coefficients in heave and sway are called $a_{33}, b_{33}, a_{22}$ and $b_{22}$ where the a's are two-dimensional added masses, and b's are two dimensional damping coefficients. 2 refers to sway and 3 refers to heave. Likewise, the wave forces on each section are called $r_{2}+h_{2}$ and $r_{3}+h_{3}$. $\mathrm{r}$ refers to Froude-Krylov forces and $\mathrm{h}$ refers to diffraction (scattering) forces. The phases of wave forces and moments are with respect to the wave crest at the midship location with a time function $\exp (i \omega t)$. $\omega$ is the circular frequency of encounter of the waves on the vehicle moving at speed $U$. The circular frequency of waves in a fixed coordinate systems is called $\omega_{0}$.

${ }_{\mathrm{p}} A_{i j}$ and ${ }_{\mathrm{p}} B_{i j}$ are the complete set of vehicle added mass and damping coefficients due to the integrals of sectional effects (These are called $A_{i j}$ and $B_{i j}$ in STF, but the altered notation is used here so the final notations can be identical after the lift forces on the fins are included). The first subscript, $i$, refers to the direction of the associated force and the second subscript, $j$, refers to the direction of the associated motion. Likewise, the total vehicle sectional wave forces resulting from an integral of the wave forces

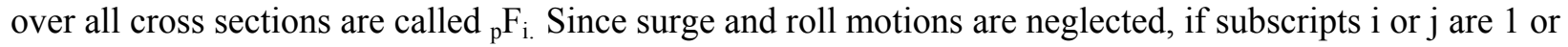
4 , the associated matrix or vector element is zero. 
Since the vehicle does not pierce the free surface, the only hydrostatic restoring forces are:

$$
{ }_{\mathrm{p}} F_{H 4}={ }_{\mathrm{p}} C_{44} \eta_{4} \text { and }{ }_{\mathrm{p}} \mathrm{F}_{\mathrm{H} 5}={ }_{\mathrm{p}} C_{55} \eta_{5} \text {, where }{ }_{\mathrm{p}} C_{44}={ }_{\mathrm{p}} C_{55}=\rho g V(G B)
$$

${ }_{\mathrm{p}} \mathrm{F}_{\mathrm{H} 4}$ is the hydrostatic roll moment (which is ignored here since roll is not considered), ${ }_{\mathrm{p}} \mathrm{F}_{\mathrm{H}}$ is the hydrostatic pitch moment, $\eta_{4}$ is the roll angle, $\eta_{5}$ is the pitch angle, $\rho$ is the water density, $g$ is the acceleration of gravity, $\mathrm{V}$ is the vehicle volume, and GB is the vertical distance that the center of buoyancy is above the center of gravity.

The contributions to vehicle damping coefficients and "springlike" restoring coefficients from lift forces on the fins are called ${ }_{\mathrm{f}} B_{i j}$, and, $C_{i j}$, and the contributions to the complex amplitudes of wave forces and moments due to lift on the fins are called ${ }_{\mathrm{f}} F_{i .}$. There is no contribution to the added masses by the lift forces on the fins. Thus the total vehicle added masses, damping coefficients, restoring force ("spring") constants and wave force complex amplitudes, called $A_{i j}, B_{i j}, C_{i j}$ and $F_{i}$, respectively, are given by:

$$
A_{i j}={ }_{\mathrm{p}} A_{i j}, \quad B_{i j}={ }_{\mathrm{p}} B_{i j}+{ }_{\mathrm{f}} B_{i j}, \quad C_{i j}={ }_{\mathrm{p}} C_{i j}+{ }_{\mathrm{f}} C_{i j}, \quad F_{i}={ }_{\mathrm{p}} F_{i}+{ }_{\mathrm{f}} F_{i}
$$

Quantities with pre-subscript "p" are called non-lifting contributions. The ${ }_{\mathrm{p}} A_{i j} \mathrm{~s}$, ${ }_{\mathrm{p}} B_{i j} \mathrm{~s}$, and ${ }_{\mathrm{p}} F_{i} \mathrm{~s}$, are taken as those derived by STF for quantities given there without the pre-subscript, except there are no transom corrections here, and the two-dimensional added masses, damping coefficients and wave force of sections are calculated by a different method.

The quantities with pre-subscript " $\mathrm{f}$ " are the sum of contributions from the time-varying lift of all fins on the vehicle. With $\mathrm{U}$ being the forward speed of the vehicle, $\mathrm{T}$ being the period of vehicle motions and $\mathrm{c}$ being a typical fin chord length, the ratio $\mathrm{c} / \mathrm{UT}<<1$ for almost all operational conditions so that quasisteady lift calculations for each fin are expected to provide good estimates.

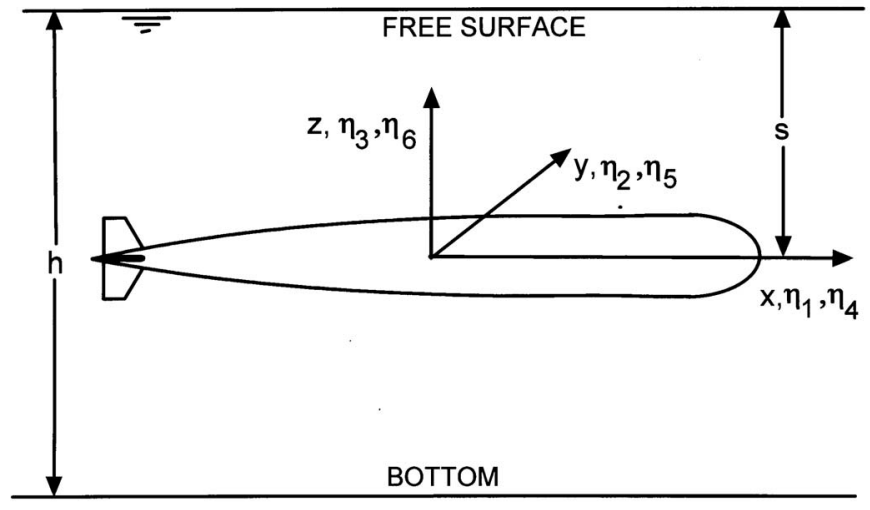

Figure 1. Coordinate and Motion Definitions

The coordinate system $(\mathrm{x}, \mathrm{y}, \mathrm{z})$, the definition of unsteady vehicle motions, and definitions of the submergence, $\mathrm{s}$, and water depth, $\mathrm{h}$, are shown in Figure 1. $\mathrm{x}$ is forward, $\mathrm{y}$ is to port and $\mathrm{z}$ is upward. The linear motions in the $\mathrm{x}, \mathrm{y}$ and $\mathrm{z}$ directions are $\eta_{1}, \eta_{2}$, and $\eta_{3}$, and the rotational motions about these axes are $\eta_{4}, \eta_{5}$, and $\eta_{6}$. Here, only $\eta_{3}$ and $\eta_{5}$ in the vertical plane and $\eta_{2}$ and $\eta_{6}$ in the horizontal plane will be considered. It will be assumed that the vehicle has left-right symmetry leading to decoupling of horizontal plane motions from vertical plane motions and that coupling effects from $\eta_{1}$ and $\eta_{4}$ are negligible. The angle of wave propagation, measured clockwise from the $\mathrm{x}$-axis viewed from above is called $\theta$. For head seas $\theta=\pi$. The relationship between fixed frame and encounter circular frequencies is:

$$
\omega=\omega_{o}-k U \cos \theta
$$

$k$ is the wavenumber, $k=2 \pi / \lambda$, where $\lambda$ is the wavelength, and $k$ and $\omega_{o}$ are related by the finite depth dispersion relation. With the water depth called $h$ this relation is:

$$
\omega_{o}^{2}=k g \tanh k h
$$




\section{Seakeeping Equations of Motion}

The forces on the vehicle, both from the sea waves and from the vehicle motions, will be linearized in the wave amplitude (called $\alpha$ ) and the vehicle motions respectively. Therefore, the response in an arbitrary sea state can be expressed in terms of the response to sinusoidal waves, over ranges of frequency and propagation angles, through the use of Fourier Integrals. This allows the equation of motion to be developed for sinusoidal waves.

The form of the equations of motion will be for all six motions to be consistent with other work, but here all terms involving surge or roll are set to zero. The complex amplitude of any sinusoidal wave is called $\alpha$, with associated force and moment complex amplitudes called $F_{j}, j=1, \ldots, 6$. The wave elevation is: $\eta=\alpha \exp (\mathrm{i} \omega \mathrm{t}-\mathrm{kx} \cos \theta+\mathrm{ky} \sin \theta)$. In water of depth $h$, the associated velocity potential is $\phi=\beta \exp (i \omega t-k x \cos \theta+k y \sin \theta) \cosh k(z+h)$. Physical quantities correspond to the real parts of these, and similar, expressions. $\alpha$ and $\beta$ are related by the free surface boundary conditions as:

$$
\beta=\frac{i \omega}{k \sinh (k h)} \alpha
$$

The linearized vehicle equations of motion in the presence of a sinusoidal wave as given by STF are:

$$
\sum_{k=1}^{6}\left[\left(M_{j k}+A_{j k}\right) \ddot{\eta}_{k}+B_{j k} \dot{\eta}_{k}+C_{j k} \eta_{k}\right]=F_{j} \exp (i \omega t)
$$

where $M_{j k}$ is the 6 x 6 mass matrix of the vehicle and $t$ is time. Equations and terms with $j$ or $k$ equal to 1 or 4 are ignored.

The equations in the vertical and horizontal planes for a vehicle with port-starboard symmetry, and with the center of gravity at $\left(\mathrm{x}_{\mathrm{c}}, 0, \mathrm{z}_{\mathrm{c}}\right)$, are decoupled from each other. The vertical plane equations, including the $\mathrm{C}_{35}$ term due to fin lift forces, but neglecting the coupling with surge are:

$$
\begin{gathered}
\left(M+A_{33}\right) \ddot{\eta}_{3}+B_{33} \dot{\eta}_{3}+\left(-M x_{c}+A_{35}\right) \ddot{\eta}_{5}+B_{35} \dot{\eta}_{5}+C_{35} \eta=F_{3} \exp (i \omega t) \\
\left(-M x_{c}+A_{53}\right) \ddot{\eta}_{3}+B_{53} \dot{\eta}+\left(I_{5}+A_{55}\right) \ddot{\eta}_{5}+C_{55} \eta_{5}=F_{5} \exp (i \omega t)
\end{gathered}
$$

In equation (7), the term involving $C_{33}$ has been omitted since it is zero for underwater vehicles. $M$ is the mass of the vehicle and $I_{5}$ is its moment of inertia in pitch. ${ }_{\mathrm{p}} C_{35}$ is zero for underwater vehicles, but ${ }_{\mathrm{f}} C_{35}$ is not zero if the vehicle has fin lift forces with vertical components.

The horizontal plane equations, but with effects of surge and roll omitted, and addition of restoring forces and moments due to fin lift forces, are:

$$
\begin{gathered}
\left(A_{22}+M\right) \ddot{\eta}_{2}+B_{22} \dot{\eta}_{2}+\left(M x_{c}+A_{26}\right) \ddot{\eta}_{6}+B_{26} \dot{\eta}_{6}+C_{26} \eta_{6}=F_{2} \exp (i \omega t) \\
\left(M x_{c}+A_{62}\right) \ddot{\eta}_{2}+B_{62} \dot{\eta}_{2}+\left(A_{66}+I_{6}\right) \ddot{\eta}_{6}+B_{66} \dot{\eta}_{6}+C_{66} \eta_{6}=F_{6} \exp (i \omega t)
\end{gathered}
$$

The terms in equations (7) through (10) involving $\mathrm{x}_{\mathrm{c}}$ do not appear in STF because in the coordinate system used there $x_{c}=0 . \mathrm{I}_{6}$ is the vehicle moment of inertia in yaw. All the B-terms are augmented by fin lift forces. The terms involving $C$ in the above two equations do not normally appear in the seakeeping equations of motion for ships. They appear here as a result of accounting for the lift forces on the fins when the vehicle has a yaw angle. Transverse velocities on fins when the vehicle has forward speed contribute to the wave forces $\left(F^{\prime} \mathrm{s}\right)$ and to the damping coefficients $\left(B^{\prime} \mathrm{s}\right)$.

The fluid mechanics part of problem is determination of the $A^{\prime} \mathrm{s}, B^{\prime} \mathrm{s}, C$ 's and $F$ 's. This is done by finding the terms that are not due to fin lift forces, as formulated in STF, except the sectional strip forces are calculated differently, and then calculating the terms due to fin lift forces. 


\section{Added Mass , Damping , and Wave Force Coefficients due to non-Lifting Effects}

\subsection{Equations relating Sectional to Total Coefficients}

As given in STF, but neglecting effects of roll and assuming the submerged vehicle ends in a section of zero area at the stern (no transom),

\begin{tabular}{|c|c|c|c|}
\hline${ }_{p} A_{33}=\int a_{33} d x$ & (11) & ${ }_{p} B_{33}=\int b_{33} d x$ & (12) \\
\hline${ }_{p} A_{35}=-\int x a_{33} d x-\frac{U}{\omega^{2}} B_{33}$ & (13) & ${ }_{p} B_{35}=-\int x b_{33} d x+U A_{33}$ & (14) \\
\hline${ }_{p} A_{53}=-\int x a_{33} d x+\frac{U}{\omega^{2}} B_{33}$ & (15) & ${ }_{p} B_{53}=-\int x b_{33} d x-U A_{33}$ & (16) \\
\hline${ }_{p} A_{55}=\int x^{2} a_{33} d x+\frac{U^{2}}{\omega^{2}} A_{33}$ & (17) & ${ }_{p} B_{55}=\int x^{2} b_{33} d x+\frac{U^{2}}{\omega^{2}} B_{33}$ & (18) \\
\hline${ }_{p} A_{22}=\int a_{22} d x$ & (19) & ${ }_{p} B_{22}=\int b_{22} d x$ & (20) \\
\hline${ }_{p} A_{26}=\int x a_{22} d x+\frac{U}{\omega^{2}} B_{22}$ & (21) & ${ }_{p} B_{26}=\int x b_{22} d x-U A_{22}$ & (22) \\
\hline${ }_{p} A_{62}=\int x a_{22} d x-\frac{U}{\omega^{2}} B_{22}$ & (23) & ${ }_{p} B_{62}=\int x b_{22} d x+U A_{22}$ & (24) \\
\hline${ }_{p} A_{66}=\int x^{2} a_{22} d x+\frac{U^{2}}{\omega^{2}} A_{22}$ & (25) & ${ }_{p} B_{66}=\int x^{2} b_{22} d x+\frac{U^{2}}{\omega^{2}} B_{22}$ & (26) \\
\hline${ }_{p} F_{3}=\rho \alpha \int\left(f_{3}+h_{3}\right) d x$ & (27) & ${ }_{p} F_{5}=-\rho \alpha \int\left[x\left(f_{3}+h_{3}\right)+\frac{U}{i \omega} h_{3}\right] d x$ & (28) \\
\hline${ }_{p} F_{2}=\rho \alpha \int\left(f_{2}+h_{2}\right) d x$ & (29) & ${ }_{p} F_{6}=-\rho \alpha \int\left[x\left(f_{2}+h_{2}\right)+\frac{U}{i \omega} h_{2}\right] d x$ & (30) \\
\hline
\end{tabular}

The integrals are over the length of the vehicle, with the effect of longitudinal slopes on the transverse forces neglected by to the slenderness approximation. The f-terms are Froude-Krylov wave forces, and the h-terms are wave forces due to the diffracted waves.

The wave excitation force (complex) amplitudes from non-lifting effects are derived by STF in terms of zero-speed integrals, over the length of the vehicle, of the sectional Froude-Krylov forces, $\left(f_{2}\right.$ and $f_{3}$ in sway and heave respectively), and integrals of the sectional diffraction forces, $\left(h_{2}\right.$ and $h_{3}$ in sway and heave respectively) plus terms due to forward speed. The Froude-Krylov forces are due to the pressure distributions in the incident wave and the diffraction forces are associated with the diffracted wave generated because the incident wave orbital velocities have components that are normal to the surface of the vehicle. The sectional forces are the twodimensional forces, per unit length for a shape equal to the cross section of the vehicle at each longitudinal location. The way the Froude-Krylov sectional forces will be computed here are the 
same as done in STF, except that the finite depth wave pressures are used here whereas STF used the infinite depth pressures. Contrary to the methods used in STF, here $\mathrm{a}_{22}, \mathrm{~b}_{22}, \mathrm{a}_{33}, \mathrm{~b}_{33}, \mathrm{~h}_{2}$, and $\mathrm{h}_{3}$ are determined by solving two-dimensional Green's theorem formulations.

\subsection{The Free Surface Boundary Condition}

The linearized three dimensional free surface boundary condition, in a reference frame moving forward at speed $U$, derived by STF and others is:

$$
\left[(i \omega-U \partial / \partial x)^{2} \phi+g \partial \phi / \partial z\right]_{z=0}=0, \quad \phi=\phi_{I}, \phi_{D}, \phi_{j}
$$

$\phi_{I}$ is the incident wave velocity potential, $\phi_{D}$ is the diffracted wave potential, and $\phi_{j}$ is the sinusoidal radiation potential for oscillation in the $j^{\text {th }}$ degree of freedom. $j=2,3,5$, or 6 are considered here. For a wave satisfying the dispersion relation (4), the frequency conversion equation (3) and having $x$ dependence $\exp (-i k x \cos \theta)$, equation (31) becomes:

$$
\left[-\omega_{o}^{2} \phi+g \frac{\partial \phi}{\partial z}\right]_{z=0}=0, \quad \phi=\phi_{I}, \phi_{D}
$$

The $x$-dependence of the linearized incident wave $\left(\phi_{I}\right)$ is exactly $\exp (-i k x \cos \theta)$ and this is approximately true for the diffracted wave $\left(\phi_{D}\right)$. Equation (32) will be used as the free surface boundary condition for these potentials. An approximation that forms the basis of strip theory is:

$\omega \phi_{j} \square U \partial \phi_{j} / \partial x$. Thus the free surface boundary condition used for the radiation potentials is

$$
\left[-\omega^{2} \phi_{j}+g \frac{\partial \phi_{j}}{\partial z}\right]_{z=0}=0
$$

Most treatises on strip theory for surface ships claim that this approximation makes strip theory strictly valid for wavelengths that are small in comparison to the length of the ship. The fact that experiments agree with theory for longer waves is attributed to the fact that hydrostatic and Froude-Krylov forces are dominant for long waves. However, for underwater vehicles, hydrostatic forces from equivalent incident waves and unsteady vehicle motions are very much smaller than for surface vessels, Yet, as will be shown subsequently here, experimental results agree with strip theory for wavelengths that are considerably longer than vehicle lengths. This leads to a more detailed analysis of the approximation to the linearized free surface boundary condition. Expanding equation (31), and assuming that $O\left[\left(\frac{\partial \phi_{j} / \partial x}{\phi_{j}}\right)_{z=0}\right] \leq \frac{1}{L}$, where $\mathrm{L}$ is the vehicle length, use of equation (33) is equivalent to:

$$
\omega^{2} \square \frac{4 U^{2}}{L^{2}} \Rightarrow \frac{4 U^{2}}{(L \omega)^{2}} \square 1
$$

The actual waves generated by an oscillating object with forward speed are extremely complicated, but the characteristic wave number in the so called "zero forward speed" problem is related to $\omega$ by the dispersion relation.

Deep Water Limit. In deep water, $\omega^{2}=g k$. Defining the Froude number as $F=U / \sqrt{g L}$, inequality (34) becomes:

$$
\lambda \square \frac{\pi L}{2 F^{2}}
$$

$\lambda$ is the wavelength generated by oscillation at the encounter frequency. A typical AUV Froude number is 0.3 . In this case, inequality (35) gives: $\lambda \square 17.5 \mathrm{~L}$. 
Shallow Water Limit. In shallow water, $\omega^{2}=g h k^{2}$ and inequality (34) becomes:

$$
\lambda \square \frac{\pi}{F} \sqrt{h L}
$$

For the operating conditions of, $h=L$ and $F=0.3, \lambda \square 10.5 L$.

Thus, although the range of validity of strip theory is proportional to $L$, it is not necessarily true that validity requires $\lambda \square L$. Considerably longer waves may result in accurate computations, especially when "engineering accuracy" allows some error.

\subsection{Boundary Conditions on the Sections and on the Domain Sides and Bottom}

A typical section for which the two-dimensional velocity potential and subsequently the pressure forces are to be computed is shown in Figure 2. It is a cross section of an AUV with a circular section hull and a sonar transducer on each side. The computational domain surrounds the cross section by a rectangle on whose top the free surface boundary condition applies, the bottom on which the boundary condition is zero normal velocity, and the two sides which are taken far enough from the cross section for the vehicle-generated flow to be only outgoing waves. The boundary condition on the sides for the velocity potential, $\phi$, to represent outgoing waves is:

$$
\frac{\partial \phi}{\partial n}=-i k \phi, \quad \text { where } n \text { is the outward normal to the sides. }
$$

Numerical testing shows that a domain width of 20 vehicle diameters is sufficient for convergence of results vs. domain width. A further increase in domain width further changes results by less than $0.5 \%$. Figure 3 shows an example of the heave force excitation magnitude, $\left|F_{3}\right|$, vs domain width in head seas on a REMUS Vehicle [8], which has a diameter of $0.19 \mathrm{~m}$, computed by methods that are subsequently described and for the conditions shown in the Figure. Results are shown for speeds of 1.0 and $2.0 \mathrm{~m} / \mathrm{s}$.

The boundary conditions on the cross section depend on the problem to be solved. In all cases, the cross sections include fins and transducers so that the non lifting diffraction and radiation forces and moments include potential flow effects of these objects.

For the heave radiation problem with unit amplitude in heave, the normal derivative of the radiation potential on the surface of the cross section is:

$d \phi / d n=\hat{k} \sqsubset \bar{n}$, where $\hat{k}$ is the unit vector in the $\mathrm{z}$ (upward) direction.

For the sway radiation problem with unit amplitude in sway, the vehicle section boundary condition is: $d \phi / d n=\hat{j} \bar{n}$, where $\hat{j}$ is the unit vector in the y (to port) direction.

For the diffraction problem in sinusoidal waves, $d \phi / d n$ on the cross section is the negative of the normal velocity distribution imposed by the wave field.

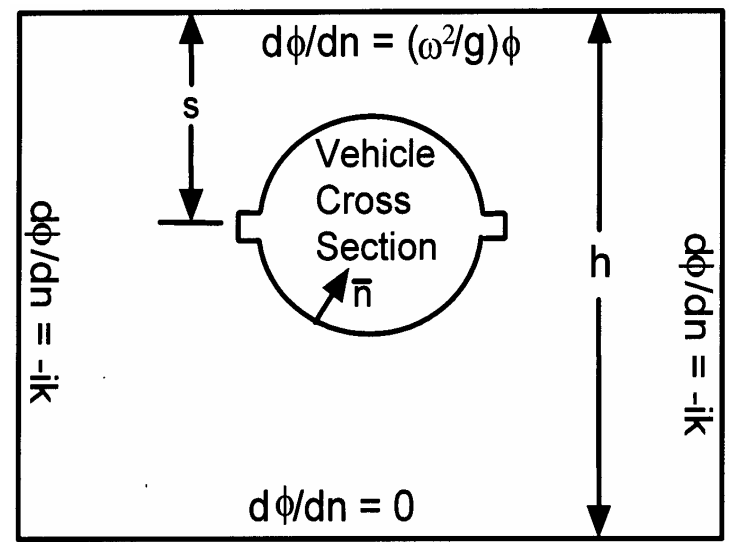

Figure 2. Cross Section of an Underwater Vehicle in a Two-Dimensional Domain. $\omega$ is changed to $\omega_{0}$ for the diffraction problem.

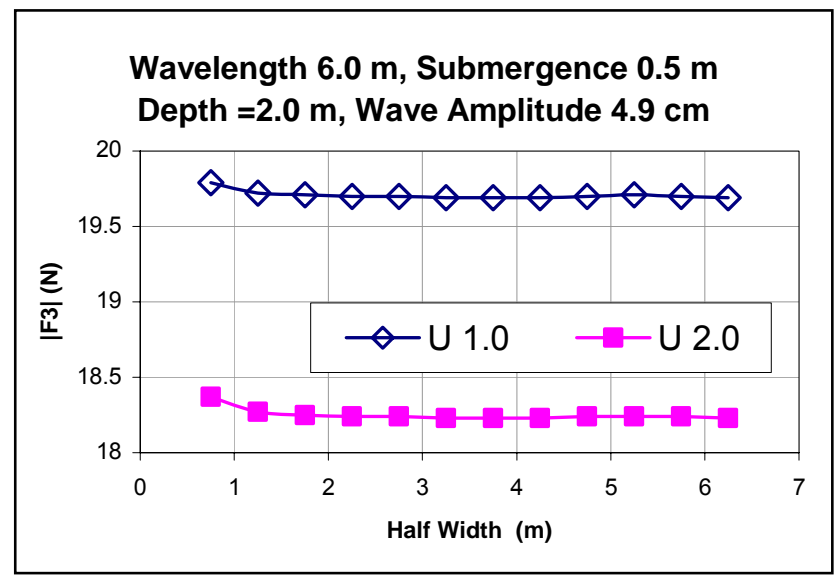

Figure 3. Convergence of Computed Heave Force Magnitude on REMUS with increasing Domain Width. -8 - 


\subsection{Calculation of Non-Lifting Two Dimensional Forces on the Sections}

With the boundary conditions specified on a section and the rectangular boundary surrounding it, Green's theorem can be used to determine the two-dimensional velocity potential and the associated forces. The Rankine Green function $G(y, z, \eta, \zeta)$ depends on the field point $(\mathrm{y}, \mathrm{z})$ and the source point $(\eta, \zeta)$ through the distance between them, $r=\left[(y-\eta)^{2}+(z-\varsigma)^{2}\right]^{1 / 2}$ and $\mathrm{G}$ is the sink potential,

$$
G=-\ln r
$$

The two-dimensional Green's theorem takes the form:

$$
\left[\int_{S} \phi(\eta, \varsigma) \frac{\partial G}{\partial n(\eta, \varsigma)}-G \frac{\partial \phi(\eta, \varsigma)}{\partial n(\eta, \varsigma)}\right] d s=\left\{\begin{array}{c}
0,(\mathrm{y}, \mathrm{z}) \text { outside } \mathrm{S} \\
-\pi \phi(y, z), \text { on } \mathrm{S} \\
-2 \pi \phi(y, z), \text { inside } \mathrm{S}
\end{array}\right\}
$$

$n$ is the normal vector drawn out of the fluid on all boundaries. S represents all the boundaries of the fluid which are a set of lines in this two-dimensional problem. $d s$ is the element of arc length on the boundaries. In all cases, $\phi$ is appended by $\exp (i \omega t)$ which is omitted for clarity. In general, $\phi$ is complex and the real part of $\phi \exp (i \omega t)$ is the physical quantity. The two-dimensional potentials are defined as $\phi_{22}$ and $\phi_{33}$ for the unit amplitude sway and heave motion problems, and as $\phi_{d}$ for the unit wave height diffraction problem.

For both the radiation problems and the diffraction problem $\partial \phi / \partial n$ is known on the vehicle crossection and on the domain bottom which is coincident with the sea bottom. As shown in Figure 2 and described in the preceding text, $\partial \phi / \partial n$ is known in terms of $\phi$ on the upper surface and on the sides of the domain. Thus, the unknowns become the values of $\phi$ over all boundaries, which are the cross section and the surrounding rectangle. Whereas the imposed heave or sway velocities are the same for all sections of the vehicle, the imposed wave velocities in the diffraction problem vary, not only around the section, but also between sections according to the wavelength and propagation angle of the wave. Zero phase of the wave is taken when the wave crest is over the center (midship) of the vehicle.

To solve equation (39) numerically, all the boundaries are divided into panels, and in this work flat panels (lines) with constant potential strength on each panel are used. In this form, the resulting twodimensional panel method becomes a set of linear equations with the number of equations and number of unknown potential strengths equal to the number of panels. Determination of the two-dimensional velocity potential by use of Green's theorem can be used for any sectional shapes.

To use equations (11) through (26) the "zero forward speed" forces, per unit length, on each section for sway and heave must be known. As described in STF, using the slender body approximation the required "zero speed" radiation forces on each section, oscillating at circular frequency, $\omega$, are determined by:

$$
\begin{gathered}
t_{j j}=-\rho i \omega \int_{C_{x}} n_{j} \phi_{j} d l \text { where } j=2 \text { for sway and } j=3 \text { for heave. } \\
t_{j j}=\omega^{2} a_{j j}-i \omega b_{j j}, \quad j=2,3
\end{gathered}
$$

$C_{x}$ is the contour around a section and $\rho$ is the fluid density. The problem is not exactly the zero forward speed problem. It is a zero forward speed problem with oscillation at circular frequency $\omega$ which depends on the forward speed. Equation (40) is constructed as the zero speed pressure force associated with the rate of change of the velocity potential, multiplied by the normal vector, and integrated around the section. The component opposed to the acceleration is due to the added mass and the component opposed to the velocity is the damping related to the energy in the outgoing waves produced when the vehicle oscillates at the wave encounter frequency. 
For the exciting forces, to use equations (27) to (30) the zero forward speed forces due to the diffracted wave and those due to the incident wave must be known. The diffracted wave is generated at circular frequency $\omega$ in the reference frame of the vehicle and the incident wave pressure is what would exist in the vehicle's absence.

$$
\begin{gathered}
h_{j}=-i \omega \int_{C_{x}} n_{j} \phi_{d} d l \text { where } j=2 \text { for sway force and } j=3 \text { for heave force. } \\
\qquad f_{j}=\frac{g}{\cosh k h} \int_{C_{x}} n_{j} \cosh k(h+z) \exp [i(-k x \cos \theta+k y \sin \theta)] d l .
\end{gathered}
$$

In equations (40) and (42), the "zero forward speed forces" are based on the zero speed potentials using the boundary conditions previously explained. Using the slender vehicle and limited wavelength approximations described above, STF, using some of the results of Ogilvie and Tuck [5] and Stokes Theorem, derived equations (40) as well as equations (11) through (30). For surface vessels, these latter equations contain an error because they neglect an integral along the waterline which is small for slender ships. In the case of underwater vehicles, this error does not occur at all. However, STF use a different approach to finding $h_{j}$ and derive an equation in which these sectional diffraction forces are proportional to $\omega_{0}$ not to $\omega$. Whether or not $\omega_{0}$ or $\omega$ should be used depends, amongst other issues, on the interpretation of the "zero forward speed force". As described previously, $\omega$ should be, and was, used in the free surface boundary condition in solving for the 2-dimensional stripwise diffraction potential. This potential has a time variation of $\exp (i \omega t)$. In calculating the pressure on the vehicle at zero forward speed, which is used in evaluating equation (42), the quantity $\omega$, as shown in equation (42) is the result of the timedependent Bernoulli equation. If the pressure on the moving vehicle is to be determined, and with a presumed spatial variation of $\exp (-i k x \cos (\theta)), \omega_{\mathrm{o}}$ rather than $\omega$ would appear in equation (42). As will be shown subsequently, the comparison between theory and experiment is superior when $\omega$ is used.

\subsection{Non-Lifting Forces on the Vehicle}

Once the two-dimensional radiation and diffraction potentials are determined by numerical solutions of equation (39) for about 35 sections along the vehicle, equations (40) through (43) can be solved and used in a numerical implementation of equations (11) through (30) to obtain the added masses, the damping coefficients, and the wave forces on the vehicle for sway, heave, pitch and yaw.

There is uncertainty about the zero forward speed sectional diffraction force which is taken here as being proportional to $\omega$ rather than $\omega_{0}$. To put this matter to rest, experimental results are compared with numerical results for $\left|\mathrm{F}_{3}\right|$ and $\left|\mathrm{F}_{5}\right|$ based on using both $\omega$ and $\omega_{o}$ in the theory. The experiments were conducted in head and stern seas in $1.52 \mathrm{~m}$ deep water on a $1.58 \mathrm{~m}$ long REMUS vehicle whose geometry is described by Von Alt et al. [8]. The experiments, which are described in detail by Sabra [9] and put in the graphical form used here by Rybka [10], covered forward speeds from 0.515 to to $2.06 \mathrm{~m} / \mathrm{s}$, wavelengths from 0.792 to $5.34 \mathrm{~m}$, and vehicle centerline submergences from 0.285 to $0.857 \mathrm{~m}$. The radius of REMUS, apart from fins and transducers is $0.095 \mathrm{~m}$.

Figure 4 is a set of graphs, for these conditions, of experiment results vs theoretical results using $\omega$ and $\omega_{o}$ in equation (42). The experimental data contains both head seas and stern seas, except for the caases of supercritical operation in stern seas when the wave speed exceeds the vehicle speed. The straight lines in the sub-figures are for the perfect correlation of Experiment vs. Theory. The correlation between theory and experiment is better when $\omega$ rather than $\omega_{o}$ is 
used in equation(42). This is in spite of the fact that $\omega_{o}$ is used in the free surface boundary condition in solving equation (39) for $\phi_{d}$, an apparent inconsistency. The reasons the superiority of using $\omega$, rather than $\omega_{o}$, in equation (42) for the underwater vehicle problem considered here, remains to be considered.

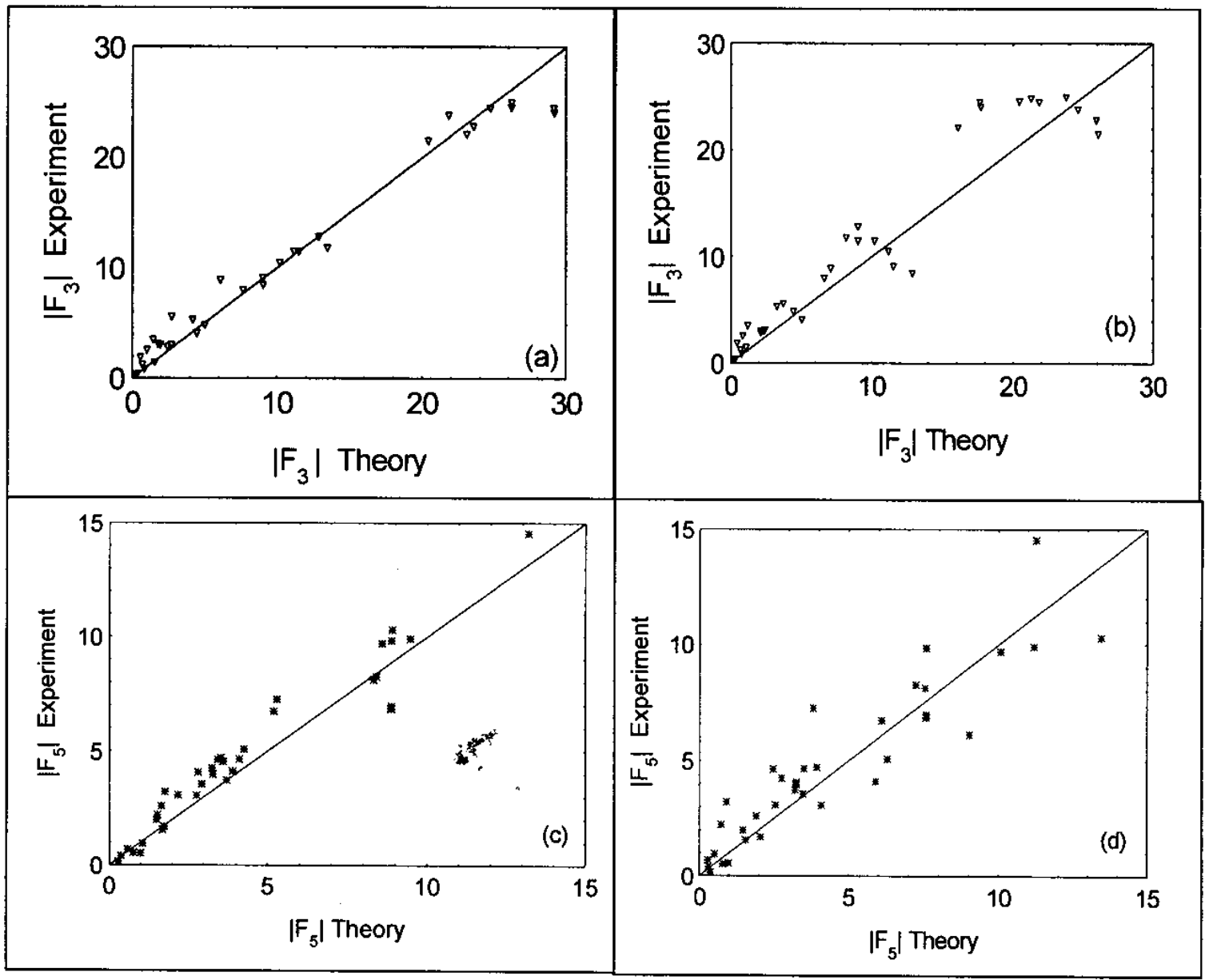

Figure 4. Comparison of Theory and Experiment for Heave Force and Pitch Moment.
(a) and (c) -- $\omega$ used in equation (42).
(b) and (d) -- $\omega_{o}$ used in equation (42).

\section{Forces and Moments Due to Lift Forces on the Fins}

\subsection{Fin Lift Force and Moment Formulations}

The general case of fin planforms at arbitrary azimuth angles results in coupling of the horizontal plane and vertical plane equations by lift forces on oblique fins. Here the case of fins whose planforms lie in horizontal and in vertical planes will be considered. 
The lift forces on fins are approximated in a quasi-static way since wavelengths that influence the vehicle are very much longer than fin chord lengths.

An approximate linearized theory for the lift force on the $m^{\text {th }}$ fin is:

$$
{ }_{f} f_{m}=\frac{1}{2} \rho U^{2} A_{m} \sigma_{m} C_{L \sigma m}
$$

where, $A_{m}$ is the effective planform area of the fin, $\sigma_{m}$ is the angle of incidence and $C_{L \sigma m}$ is the lift coefficient per unit angle of attack (in radians).

For vertical fins the yaw moment from ${ }_{f} f_{m}$ is:

$$
{ }_{f} f_{6 m}=f_{m} x_{m}
$$

and for horizontal fins the pitch moment from the fin is:

$$
f_{5 m}=-{ }_{f} f_{m} x_{m} \text {. }
$$

$x_{m}$ is the longitudinal location of the center of lift force of the fin, usually approximated at the mean quarter chord of the fin.

The linearized angle of incidence is the crossflow, $V_{\mathrm{m}}$, generated by vehicle motions and sea waves divided by the forward speed, $U$, plus the unsteady angle of the vehicle. Thus the equation for the lift force on a single fin becomes:

$$
{ }_{f} f_{m}=\frac{1}{2} \rho V_{m} U A_{m} C_{L \sigma m}+\frac{1}{2} \rho U^{2} A_{m} \sigma_{T m} C_{L \sigma m}
$$

$\sigma_{T m}$ is the vehicle angle, pitch or yaw, which imposes an angle of incidence on the $m^{\text {th }}$ fin. The first term in equation (47) is proportional to unsteady velocities, $V_{m}$, so it contributes either to wave forces or damping forces, whereas the second term is proportional to unsteady vehicle angles so it contributes to restoring "spring" constants in the equations of motion.

For any fin, or set of fins, it is necessary to estimate the effective area, $\mathrm{A}_{\mathrm{m}}$, the center of fin lift force, $x_{m}$, and $C_{L \sigma m}$. The experiments, whose results will be compared with the theory, were done on a REMUS class vehicle (Von Alt, et al., [8]) which has four identical tail fins in a cruciform shape. A drawing of REMUS, viewed from the side, is shown in Figure 5. The spans, perpendicular to the hull, of the sonar transducers and ballast bracket are so small that their lift force is negligible. Their influence on the potential flow added mass, damping coefficients and wave forces is included since they are part of the vehicle shape used in solving the potential flow problem. Figure 6 shows details of the vertical planform of the tail fins. The horizontal tail fin is identical. Experiments by Oller [11] have shown that the effective vertical tail fin of REMUS is comprised of the two vertical fins and the section of the vehicle centerplane formed by connecting the fin root leading edges and the fin root chord leading edges and the fin root trailing edges as shown in Figure 7. This effective fin has an area of $0.02 \mathrm{~m}^{2}$, a mean longitudinal quarter chord location with respect to the vehicle midship of $x=-0.7$ meters and aspect ratio of 3.23. For aspect ratios (AR) between 3 and 5, Hoerner and Borst [12] predict values of $C_{L \sigma}$, with their formula converted from degrees to radians, as:

$$
C_{L \sigma}=\left[0.175+\frac{0.175}{(A R)^{2}}+\frac{0.454}{(A R)}\right]^{-1}
$$




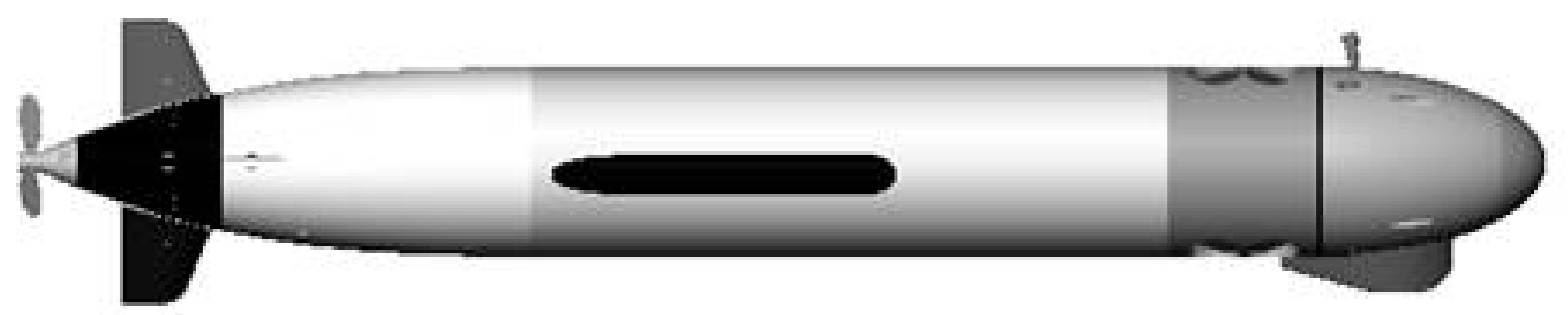

Figure 5. Side View of the REMUS Vehicle.

The vehicle length is 1.58 meters.

It was tank tested and seakeeping computations were done without the propeller.

Equation (48) is developed for straight wings with rounded tips, but similar results are found for other planforms [12] so that equation (48) can be used for most vehicle fin planforms.

The experimental values of $C_{L \sigma}$ found by Oller [11] are nearly independent of vehicle submergence and have an average value of 3.04 in good correspondence with equation(48). For the REMUS equivalent tail fins, each with aspect ratio 3.23 , equation (48) results in $C_{L \sigma}=3.01$.

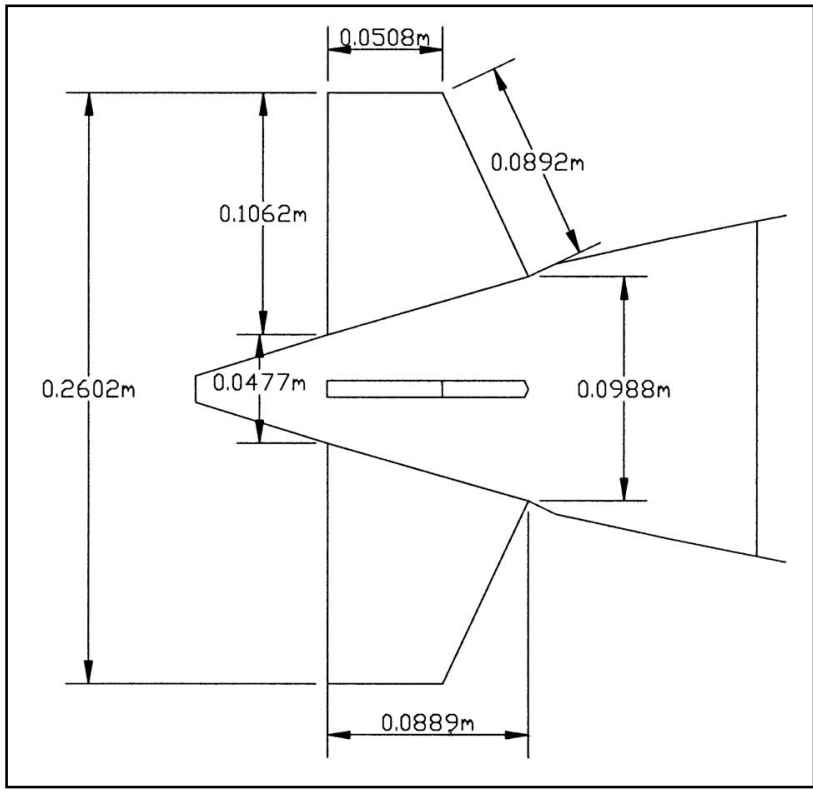

Figure 6. Vertical Profile of the Tail Section of the

REMUS Autonomous Underwater Vehicle

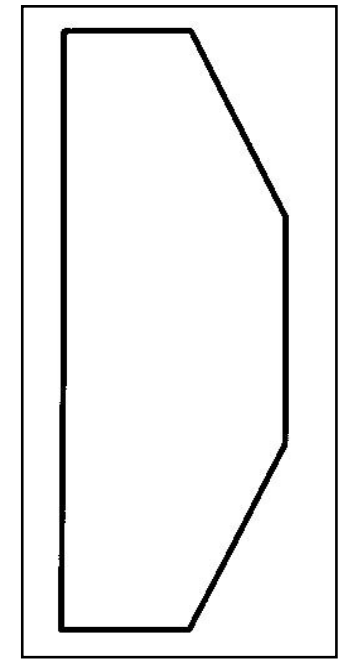

Figure 7. Equivalent Fin.

\subsection{Wave Forces and Moments Due to Fin Lift}

In water of depth $h$, the linearized wave elevation, $\varsigma$, for a propagation angle $\theta$, measured clockwise from the positive $\mathrm{x}$-axis, and encountered from the moving vehicle is:

The related velocity potential is:

$$
\varsigma=\alpha \exp [i(\omega t-k x \cos \theta+k y \sin \theta)]
$$

$$
\phi=\alpha \frac{i \omega_{o}}{k \sinh k h} \exp [i(\omega t-k x \cos \theta+k y \sin \theta)] \cosh k(h+z)
$$


For this wave, at location $(x, y,-s)$ the vertical, positive upward, wave-induced fluid velocity, $w$, is:

$$
w=\frac{i \omega_{o} \alpha}{\sinh k h} \exp [i(\omega t-k x \cos \theta+k y \sin \theta)] \sinh k(h-s)
$$

and the $y$-directed wave-induced horizontal fluid velocity, $\mathrm{v}$, is:

$$
v=\frac{-\omega_{o} \alpha \sin \theta}{\sinh k h} \exp [i(\omega t-k x \cos \theta+k y \sin \theta)] \cosh k(h-s)
$$

$\omega_{o}$ is the circular frequency of the wave in a fixed frame of reference.

Vehicles whose fins line in vertical and horizontal planes usually have their planes in symmetrical pairs. For example, if a vehicle has a vertical fin above its centerline, it will usually have a mirror image vertical fin below the centerline as shown in Figure 5. The vertical center of lift of the combination of fins and the lifting portion of the vehicle between them is at the centerline submergence for a vehicle with updown hull symmetry. Furthermore, the length scales of the fins are generally much smaller than the wavelengths of sea waves. As a result, the value of s in equations (51) and (52) can be taken as the centerline submergence of the vehicle.

Similarly, horizontal planes generally exist in symmetrical pairs so the value of y in equations (51) and (52) can be taken as zero. Some underwater vehicles have three fins in a cross plane and these require a modification to what is presented here.

The fin lift forces due to sea waves come only from wave-induced angles of attack so they are described by just the first term on the right hand side of equation (47). Thus, linearized wave forces and moments due to lift forces on the fins are:

$$
\begin{gathered}
{ }_{f} F_{2}=\sum_{m-\text { Vertical }} \frac{1}{2} \rho U A_{m} C_{L \sigma m} \frac{-\omega_{o} \alpha \sin \theta_{m}}{\sinh k h} \exp \left[i\left(\omega t-k x_{m} \cos \theta_{m}\right)\right] \cosh k(h-s) \\
F_{3}=\sum_{m-\text { Horizontal }} \frac{1}{2} \rho U A_{m} C_{L \sigma m} \frac{i \omega_{o} \alpha}{\sinh k h} \exp \left[i\left(\omega t-k x_{m} \cos \theta_{m}\right] \sinh k(h-s)\right. \\
{ }_{f} F_{5}=\sum_{m-\text { Horizontal }}-\frac{1}{2} \rho U A_{m} C_{L \sigma m} x_{m} \frac{i \omega_{o} \alpha}{\sinh k h} \exp \left[i\left(\omega t-k x_{m} \cos \theta_{m}\right] \sinh k(h-s)\right. \\
{ }_{f} F_{6}=\sum_{m-\text { Vertical }} \frac{1}{2} \rho U A_{m} C_{L \sigma m} x_{m} \frac{-\omega_{o} \alpha \sin \theta_{m}}{\sinh k h} \exp \left[i\left(\omega t-k x_{m} \cos \theta_{m}\right)\right] \cosh k(h-s)
\end{gathered}
$$

$m$-Vertical is the group of fins with vertical planforms and $m$-Horizontal is the group of fins with horizontal planforms.

\subsection{Damping Coefficients Due to Fin Lift}

When a vehicle with forward speed $\mathrm{U}$ has unsteady vertical motion $\eta_{3}=\mathrm{N}_{3} e^{i \omega t}$, in its own reference frame it experiences vertical velocity $-\dot{\eta}_{3}=-i \omega \mathrm{N}_{3} e^{i \omega t}$, so that the complex amplitude of the linearized angle of incidence on horizontal fins is $-i \omega \eta_{3} / U$. This generates heave force and pitch moment contribution from lift on horizontal fins which are proportional to the heave velocity. Thus, they are

damping forces and moments. Using the first term on the right hand side of equation (47) for the fin lift, the damping coefficients from the lift on all horizontal fins are:

$$
{ }_{f} B_{33}=\sum_{m-\text { Horizontal }} \frac{1}{2} \rho U A_{m} C_{L \sigma m}, \quad{ }_{f} B_{53}=\sum_{m-\text { Horizontal }}-\frac{1}{2} \rho U A_{m} C_{L \sigma m} x_{m}
$$


${ }_{f} B_{33}$ is always positive, but ${ }_{f} B_{53}$ can be positive or negative, depending on the sign of $x_{m}$.

Similarly the sway damping coefficients due to sway motion induced velocities on vertical fins are:

$$
{ }_{f} B_{22}=\sum_{m \text {-Vertical }} \frac{1}{2} \rho U A_{m} C_{L \sigma m}, \quad{ }_{f} B_{62}=\sum_{m-\text { Vertical }} \frac{1}{2} \rho U A_{m} C_{L \sigma m} x_{m}
$$

For pitch motion, $\eta_{5}=\mathrm{N}_{5} e^{i \omega t}$, the vertical velocity of a fin at longitudinal position $x_{m}$ is: $-i \omega x_{m} \mathrm{~N}_{5} e^{i \omega t}$. This leads to:

$$
{ }_{f} B_{55}=\sum_{m-\text { Horizontal }} \frac{1}{2} \rho U x_{m}^{2} A_{m} C_{L \sigma m}, \quad{ }_{f} B_{35}=\sum_{m-\text { Horizontal }}-\frac{1}{2} \rho U x_{m} A_{m} C_{L \sigma m}
$$

and the damping coefficients due to yaw are:

$$
{ }_{f} B_{66}=\sum_{m-\text { Vertical }} \frac{1}{2} \rho U x_{m}^{2} A_{m} C_{L \sigma m}, \quad{ }_{f} B_{26}=\sum_{m-\text { Vertical }} \frac{1}{2} \rho U x_{m} A_{m} C_{L \beta m}
$$

\subsection{Restoring Force Constants Due to Fin Lift}

When the vehicle has a non-zero yaw angle, $\eta_{6}$ fins with vertical planforms have an associated angle of incidence, and when there is a non-zero pitch angle, $\eta_{5}$, fins with a horizontal planforms have an associated angle of attack. This causes fin lift through the second term on the right hand side of equation (47). Since the lift forces are proportional to yaw or pitch angle, they can be described restoring force "spring" constants, $\mathrm{C}_{26}, \mathrm{C}_{66}, \mathrm{C}_{35}$, and $\mathrm{C}_{55}$. These are given by:

$$
\begin{aligned}
C_{35} & =\sum_{m \text {-Horizontal }} \frac{1}{2} \rho U^{2} A_{m} C_{L \sigma m}, & C_{55} & =\sum_{m-\text { Horizontal }}-\frac{1}{2} \rho U^{2} A_{m} x_{m} C_{L \sigma m} \\
C_{26} & =\sum_{m \text {-Vertical }}-\frac{1}{2} \rho U^{2} A_{m} C_{L \sigma m}, & C_{66} & =\sum_{m-\text { Vertical }}-\frac{1}{2} \rho U^{2} A_{m} x_{m} C_{L \sigma m}
\end{aligned}
$$

\section{Validation of Strip Theory for Underwater Vehicles}

\subsection{Numerical Implementation of the Theory}

The theory was numerically implemented by starting with the cross section panel method briefly described previously. Rybka [10] describes the number of panels and their distribution on the external boundary required for numerical convergence. Cross section number and spacing depends on the vehicle shape. The external transducers and fins usually determine the number of cross sections required to specify the vehicle shape since the hulls are slender. Usually 20 unequally spaced cross sections capture the vehicle shape and wave variations along the length sufficiently well. Numerical convergence in the solution to equation (39) is typically achieved with 16 panel segments on the basic hull, four panels on each side of each fin, one or two panels along each side of each transducer, and one or two panels on the fin tips or on the outermost part of each transducer. Due to the unequal spacing between cross sections, the integrals in equations (11) through (30) are done with the Trapezoidal Rule. The contributions to the vehicle force coefficients from fin lift forces were calculated according to equations (57) through (62), and added to the potential flow force coefficients according to equations (2). 


\subsection{Computational Verification}

Rybka [10] has done a thorough comparison of the results of the Strip Theory, at wave angles of 0,45 , 90, 135 and 180 degrees, with results from the fully three dimensional computer program WAMIT [13] which deals with the zero forward speed linearized wave and motion forces on an object in the ocean. WAMIT, developed over many years under the supervision of Professor John Nicholas Newman, has been fully verified for numerical accuracy and convergence. Outstanding correspondence for diffraction (magnitude and phase) and radiation force results between the two programs was obtained except for the diffraction pitch and yaw moment magnitudes in beam seas, $\theta=\pi / 2$ (90 degrees), where differences of $30 \%$ occur. Figure 8 shows a typical comparison for all other wave angles. It is for diffraction sway forces for 21 computer runs of both the Strip Theory and WAMIT on the REMUS vehicle with a wave angle of 135 degrees (bow quartering seas) and water depths of 1.52 and $5.0 \mathrm{~m}$, submergences varying between 0.476 and $2.0 \mathrm{~m}$, and wavelengths varying between 5.0 and $30.0 \mathrm{~m}$. Each of the 21 runs has a distinct set of parameter values in the ranges and depths given above. The straight lines in the sub-figures indicate perfect correspondence. For the phase, many points are on top of each other. Importantly, there are no first order fin lift forces at zero forward speed so this comparison does not include a major component of this Strip Theory.

|F2|, Wave Angle $=135$

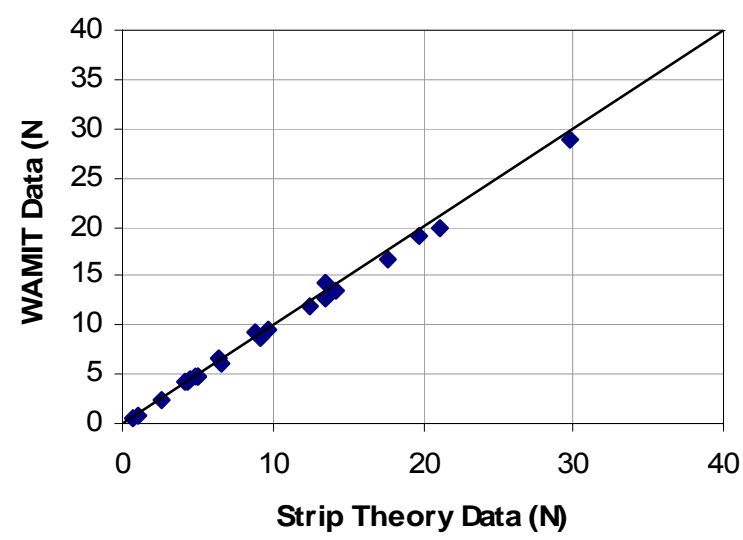

Phase F2, Wave Angle = 135

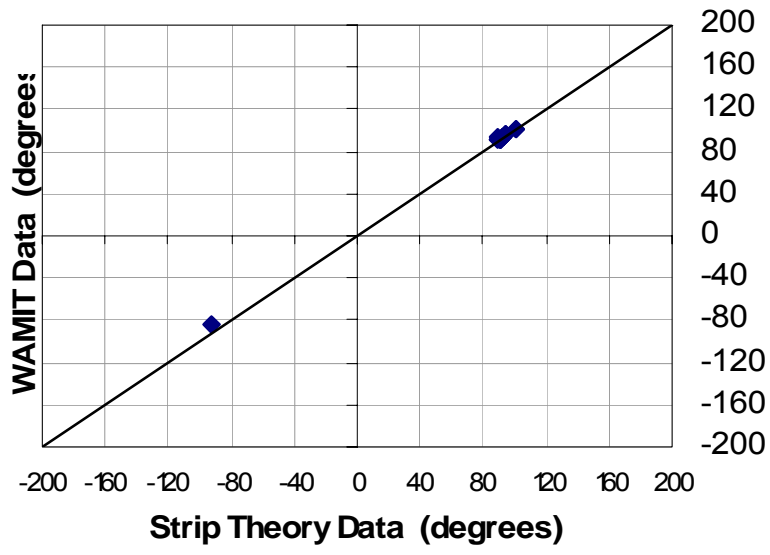

Figure 8. Computational Comparison of Magnitude and Phase of $F_{2}$ at Zero Speed

\subsection{Experiments}

Two sets of experiments were conducted. In each of them a 6-axis force and moment transducer was mounted to a bulkhead inside the vehicle. A strut which was connected to the mechanism that moved the vehicle was attached to the transducer by passing through a small hole in the top of the hull. The first set of experiments was for measuring the wave forces and moments on a steadily moving full size $1.58 \mathrm{~m}$ long REMUS vehicle [8] in the smaller of the two wave/towing tanks at the U.S. Naval Academy with a water depth of $1.52 \mathrm{~m}$. The vehicle centerline submergence was varied between 0.285 and $0.875 \mathrm{~m}$, the vehicle speeds between 0 and $2.06 \mathrm{~m} / \mathrm{s}$, and the wave lengths between 0.792 and $6.340 \mathrm{~m}$. The experimental wave amplitudes between 0.006 and $0.054 \mathrm{~m}$ were half of the planned amplitudes due to the tank operators interpreting specified amplitudes as wave heights. The largest specified amplitudes were based on the capabilities of the wave maker in the tank. Waves were measured by a moving wave staff attached to the tow carriage and the measured wave phase was shifted theoretically to account for the longitudinal distance between the wave staff and the vehicle midship. With forward speed, the geometry of the tank required head seas or stern seas. At zero speed all wave angles could be tested. Analyses for 
the amplitude and phase of waves and forces [9,11] were done by taking Fourier transforms of the measured data. To have wave frequency correspond to one frequency in a Fast Fourier Transform (FFT), it was important to select a data segment with an integer number of cycles. This was an imprecise process because of noisy data. The results showed repeatability of resulting amplitudes, but force phase estimates for identical conditions varied by up to \pm 0.7 radians ( 40 degrees). For a few points the theory vs experiment phase differences were even larger. Most of the phase difference is attributed to experimental error. This difference did not appear in the Strip Theory vs WAMIT comparisons shown by Rybka [10].

A measure of the accuracy of the wave force data is provided by 25 pair of the data runs ( 50 runs) where for each pair all parameters were identical except for the wave amplitude which varied by a factor between 1,5 and 2. Since the forces were found to be linear in wave amplitude, experimental accuracy can be, at least partially, assessed by comparing the ratios of force or moment divided by wave amplitude, yielding normalized forces and moments, in each of these pair of runs. For zero error, these pair of rations would be identical. In other words, the normalized ratios of forces an moments for each pair of runs would be 1.0. For all 25 pair of runs, the RMS values of the deviation from unity of these ratios were $7.3 \%$ for the forces and $10.4 \%$ for the moments. Again, for zero error the phases of the forces and moments with r4espect to the wave phase would be identical. For six pair of the runs, the phases differed by more than a few degrees. However, in each of these six pair of runs, the difference in force phases was within a few degrees of the difference between moment phases. This is indicative of an error in assessing the phase of the wave elevation. Of the remaining 19 pair of runs, the RMS value of the deviation of the phases from identical was 8.8 degrees for the forces and 7.3 degrees for the moments. The wave force experiments are considered to be of excellent quality

The second set of experiments [11], for forces induced by vehicle oscillations in the presence of forward speed, was conducted on an 0.4334 scale model of REMUS in a tank with a programmable 5-axis gantry above and in the tank at the Massachusetts Institute of Technology (MIT). Building the gantry, and writing the software to operate the gantry, accept force transducer output through an analog to digital converter, a PC computer and give synchronized output of forces and motions was done by Mr. Paul D'Ambra of D'Ambra Technologies, Inc. in Andover Massachusetts. Inertial forces due to the mass of the model vehicle were subtracted from the measured forces to obtain the hydrodynamic forces and moments on the vehicle. Many problems occurred with the gantry hardware and its operating and measurement software, largely because operational frequencies were higher than those the hardware and software of the gantry were designed and built to provide. During the two-year period of this portion of the project, while much data were collected, continuous improvement of the gantry hardware and software, were conducted Paul D'Ambra. His unfortunate death ended the improvements. As a result only the 20 final experimental runs, five for each of the motions of sway, heave, yaw and pitch, out of the several hundred runs conducted, are the most accurate, but their data must still be considered qualitative. It is important; nevertheless, because, when the vehicle has forward speed, the data confirm the theoretical finding that fin lifting forces dominate damping coefficients and generate restoring "spring" constants.

\subsection{Comparison of StripTheory and Experimental Results}

The wave forces, magnitudes and phases, of the REMUS vehicle, were extracted from the measured data by Sabra [9], and these were compared with results of the Strip Theory which includes the influence of fin lift by Rybka [10]. Although there is noticeable scatter in the experimental results, generally good agreement was found between the results of Strip Theory and the experiments, especially for force and moment magnitudes. The only cases of serious disagreement are the zero speed pitch and yaw moment magnitudes from beam seas, $\theta=\pi / 2$ radians, for which the theory gives values that are about $33 \%$ of the experimental measurements. Since the beam sea pitch and yaw moments, in a coordinate system whose origin is centered on the vehicle length, result from deviation in fore and aft symmetry, they are 
very small; about $7 \%$ of the head and stern sea pitch moments in the same waves. Thus the numerical difference between Strip Theory and experimental pitch and yaw moments in beam seas is small in comparison to head and stern sea pitch moments. Furthermore, tank wall effects on forces and moments are largest for zero speed. It is possible that the still small wall effects are not noticeable in the larger fundamental sway and heave forces, but significant in comparison rto the very small beam sea sway and pitch moments. Figures 9 and 10 show the comparison between the Strip Theory and experimental heave force, $F_{3}$, in head seas $(\theta=\pi$ radians) and with the vehicle having forward speed. The other theoretical vs experimental wave force and moment comparisons, except for the beam sea pitch and heave moment amplitudes, are similar and given by Rybka [10]. Strip Theory including the influence of fin lift is shown in the Figures by triangles, and the Strip theory without fin lifting influences is shown by squares. The parameter ranges are given above. The exact combinations of these parameters used for each run are given by Sabra [9].

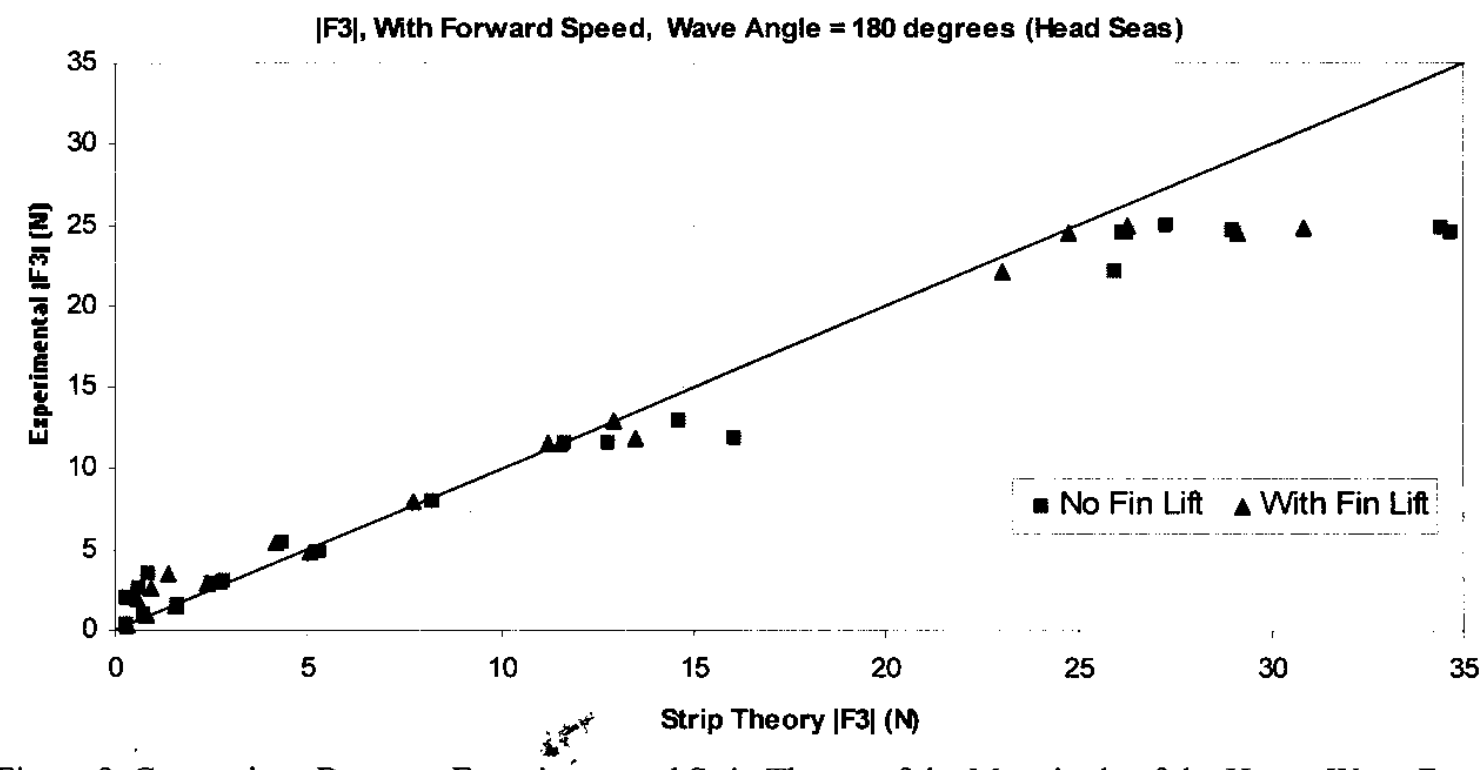

Figure 9. Comparison Between Experiment and Strip Theory of the Magnitude of the Heave Wave Force 


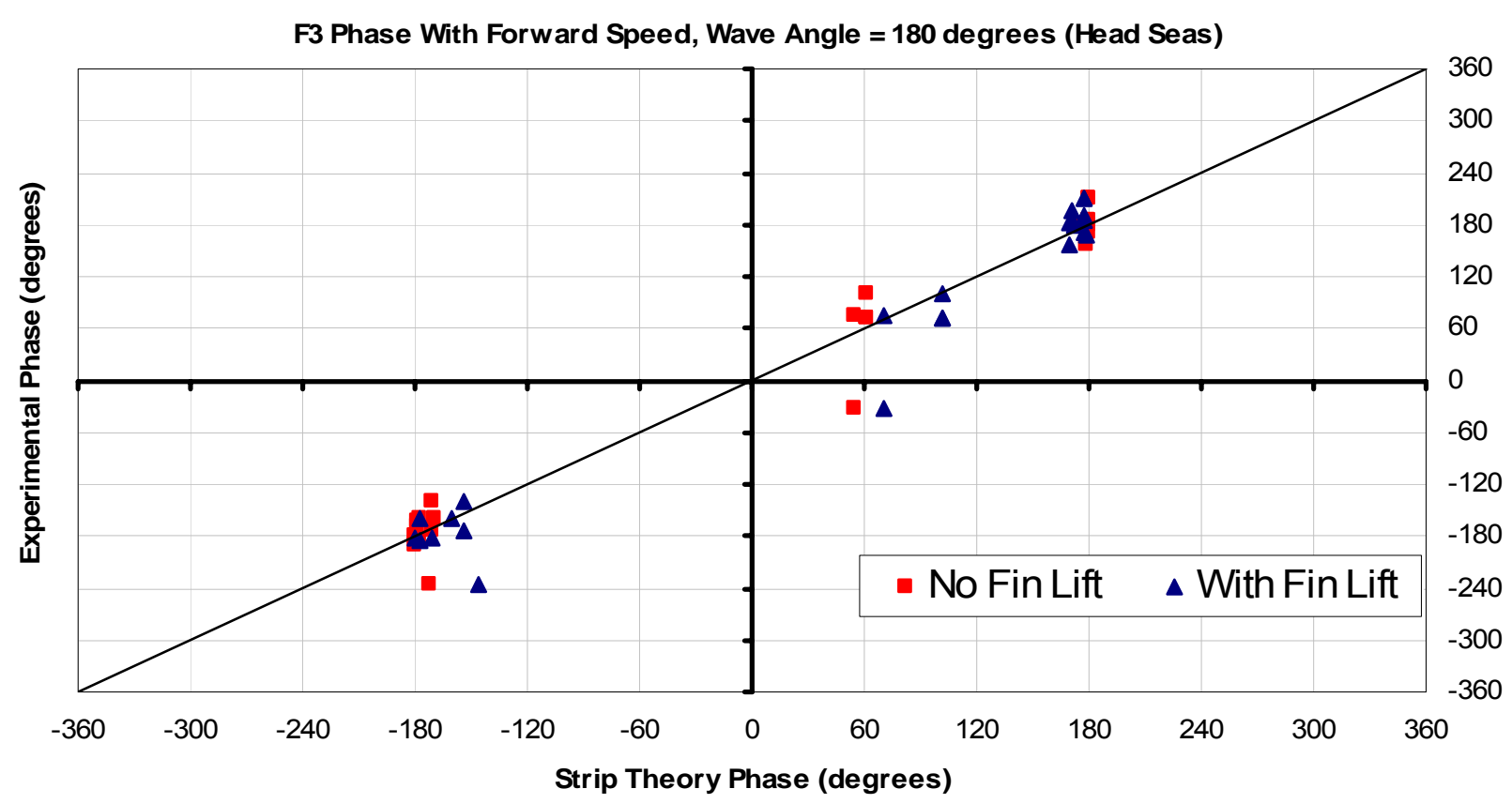

Figure 10. Comparison Between Experiment and Strip Theory of the Phase of the Heave Wave Force

Each experimental data point in Figures 9 and 10 appears twice, once as a square, compared to Strip Theory without fin lift, and once as a triangle, compared with the Strip Theory that includes fin lift forces. These two points are on a horizontal line. For $\left|F_{3}\right|$ including the lift forces improves the theory vs experiment correspondence, but for phase of $\left(\mathrm{F}_{3}\right)$ including fin lift improves correspondence in most cases and degrades correspondence in 8 cases. However, the nature of the experiments was such that magnitudes were determined much more accurately than phases. In view of this, Figure 9 shows, unambiguously, the need to include fin lifting forces in the theory, at least for relative fin area as large as on REMUS. Results similar to Figures 9 and 10 were found for all of the wave forces and moments in the experiments except in stern seas with enough forward speed for $\omega$ to be negative or for $4 \mathrm{U}^{2} /(\mathrm{L} \omega)^{2}>7$.

Table 1. Experimental and Strip Theory Force Coefficients for Vehicle Yaw Oscillatory Motion

\begin{tabular}{|c|c|c|c|c|c|c|c|c|r|r|r|r|r|r|r|}
\hline \multicolumn{2}{|c|}{ Experiments, Yaw Motion Amplitude $=10$ deg } & \multicolumn{3}{|c|}{ Theory - No Fin Lift } & \multicolumn{4}{|c|}{ Theory with fin Lift } \\
\hline $\mathrm{s}$ & $\mathrm{U}$ & $\omega$ & Yaw-M & Phs & A66 & B66 & Yaw-M & Phs & A66 & B66 & Yaw-M & Phs & B66 & A66 & C66 \\
\hline$(\mathrm{m})$ & $(\mathrm{m} / \mathrm{s})$ & $(\mathrm{rad} / \mathrm{s}$ & $(\mathrm{N}-\mathrm{m})$ & $(\mathrm{deg})$ & $\left(\mathrm{Kg}-\mathrm{m}^{2}\right)$ & $(\mathrm{N}-\mathrm{m}-\mathrm{s})$ & $(\mathrm{N}-\mathrm{m})$ & $(\mathrm{deg})$ & $\left(\mathrm{Kg}-\mathrm{m}^{2}\right)$ & $(\mathrm{N}-\mathrm{m}-\mathrm{s})$ & $(\mathrm{N}-\mathrm{m})$ & $(\mathrm{deg})$ & $(\mathrm{N}-\mathrm{m}-\mathrm{s})$ & $\left(\mathrm{Kg}-\mathrm{m}^{2}\right)$ & $(\mathrm{N}-\mathrm{m})$ \\
\hline 0.293 & 0.333 & 7.517 & 0.814 & -32 & 0.070 & 0.325 & 1.219 & -1 & 0.123 & 0.018 & 1.211 & -11 & 0.183 & 0.120 & 0.19 \\
\hline 0.293 & 1.000 & 7.517 & 1.114 & -34 & 0.093 & 0.477 & 1.738 & -1 & 0.176 & 0.027 & 1.599 & -25 & 0.524 & 0.146 & 1.68 \\
\hline 0.584 & 0.333 & 2.531 & 0.130 & -21 & 0.108 & 0.106 & 0.204 & -1 & 0.183 & 0.005 & 0.187 & -24 & 0.170 & 0.154 & 0.19 \\
\hline 0.584 & 0.333 & 7.517 & 0.828 & -32 & 0.071 & 0.336 & 2.382 & 0 & 0.127 & 0.001 & 2.357 & -10 & 0.166 & 0.124 & 0.19 \\
\hline 0.293 & 0.667 & 2.531 & 0.350 & -23 & 0.289 & 0.306 & 0.404 & -1 & 0.362 & 0.010 & 0.312 & -29 & 0.341 & 0.245 & 0.75 \\
\hline 0.584 & 1.000 & 4.980 & 0.497 & -28 & 0.102 & 0.267 & 1.245 & -1 & 0.259 & 0.012 & 0.941 & -28 & 0.509 & 0.191 & 1.68 \\
\hline
\end{tabular}

Table 1 shows yaw motion parameters and a few results for the $43.34 \%$ scale model of REMUS with a water depth of $0.795 \mathrm{~m}$. The experimental results are rough, so that the experimental yaw moment, its phase, $\mathrm{A}_{66}$ and $\mathrm{B}_{66}$ are not closely matched by the Strip Theory, either with or without fin lift. However, the fin lift brings the results of theory closer to experimental values. Importantly, the yaw moment phase and $\mathrm{B}_{66}$ from theory without fin lift are extremely small. Fin lift makes the moment phase and $\mathrm{B}_{66}$ closer to the experiments in all cases. Dominance of fin lift on damping coefficients from other motions was found as well. In view of experimental difficulties, the results of Strip Theory with fin lift are likely to be 
more accurate than the experiments. For $\omega=2.531 \mathrm{~s}^{-1}$, the zero forward speed "typical" wavelength given by the dispersion relation $\omega^{2}=k g$ tanh $k h$ is $6.33 \mathrm{~m}$ which is considerably larger than the model scale vehicle length of $0.685 \mathrm{~m}$. The phase speed of these waves is $2.55 \mathrm{~m} / \mathrm{s}$ which is large in comparison to the values of $U$ tested for this frequency so that $\omega \approx \omega_{0}$.

Without fin lift, the usual strip theory gives zero for the restoring moment "spring" constant, $C_{66}$. However with aft vertical fins on REMUS Strip Theory with fin lift yields positive values of $C_{66}$. The real part of the yaw moment divided by the yaw motion amplitude in radians is $\omega^{2} A_{66}-C_{66}$. If the entire real part of the yaw moment is attributed to $A_{66}$ and $C_{66}$ is taken as zero, as was done in analyzing the experiments, the value of the apparent yaw added mass will be $A_{66 \mathrm{c}}=A_{66}-C_{66} / \omega^{2}$. Values of $A_{66 \mathrm{c}}$ determined from the Strip theory with fin lift are shown in Table 1. The experimental values of $A_{66}$ differ less from theoretical $A_{66 \mathrm{c}}$ values than they do from the theoretical $A_{66}$ values which are independent of fin lift. This is an important finding.

\section{Computation Time}

Fast and efficient computation was obtained by developing the programs in MATLAB, optimizing them, and compiling them into executable modules using the MATLAB Compiler. This converts the MATLAB m-files into equivalent source code in the $\mathrm{C}$ programming language and then compiles the $\mathrm{C}$ source code into the executable modules. The process from the $\mathrm{m}$-files to the executable modules is "transparent" to the user. For each set of conditions, if many conditions are computed at the same time, the run time for the diffraction program is 0.2 seconds on a $3 \mathrm{GHZ} \mathrm{PC}$. This computing time is for the REMUS vehicle, using 36 cross sections with between 16 and 44 panels per section, depending on fin and transducer details. The radiation problems have not yet been optimized and compiled so the computing time in MATLAB is about 45 seconds for each set of conditions. However, the computing steps for each of the horizontal and vertical plane radiation problems is nearly the same as for the diffraction problem, so the optimized and compiled radiation code is expected to run in 0.6 seconds for each set of conditions.

\section{Conclusions}

Strip Theory, including the effects of finite depth and lift forces on fins is a fast-computing and accurate way to predict the unsteady seakeeping forces on streamlined underwater vehicles close enough to the surface to be influenced by sea waves. With these forces known, the frequency responses of vehicle motions due to sinusoidal waves of specified amplitude, frequency and phase, can be computed by the algebraic equations provided by Rybka [10] (section 8.2). These results are suitable for random wave simulations [3].

The comparison between force and moment magnitudes of numerically implemented theory and experiment is good in most conditions in the sense that errors are about the same relative size as in many comparisons between theory and experiment for strip theory applied to ships. In a large data set of REMUS diffraction forces with magnitudes between 0 and $35 \mathrm{~N}$ and moment magnitudes between 0 and $25 \mathrm{~N}$-m, typical magnitude errors are on the order of 0 to $1 \mathrm{~N}$ for forces and 0 to $0.5 \mathrm{~N}$-m for moments, except that the Strip Theory and experiment differ in beam seas and in stern seas with large enough forward speed for $\omega$ to be negative or for $4 \mathrm{U}^{2} /(\mathrm{L} \omega)^{2}>7$. Evidently, this parameter influences the diffraction problem as well as the radiation problem. Differences between theory and experiment for magnitudes of beam sea pitch and yaw moment magnitudes are large, with differences up to $4 \mathrm{~N}$-m. Part of this is experimental error, but much of it must be attributed to failure of the theory to predict accurate results in beam seas. None of this larger error appears in Rybka's comparisons for quartering seas $(\theta=45$ and 135 degrees), so the larger error must be limited to a small wave propagation angle range centered on 90 degrees. 
The difference between theoretical and experimental diffraction force and moment phases typically varies between 0 and 40 degrees and is larger for a few data points. This appears to be due to experimental error, but even if the theory yields erroneous wave force phases, it will not influence moment simulations since each sinusoidal component is assigned a random phase.

A significant contribution of this Strip Theory is inclusion of fin lift forces. They generate restoring forces and moments for motions in pitch and yaw, they generally dominate all vehicle motion damping coefficients, and they significantly influence wave forces.

\section{Acknowledgements}

The author wishes to acknowledge the wonderful contributions to the experiments and their analyses conducted for the background to this paper by the MIT graduate students, LT Gregory Sabra and LT Jan Rybka of the U.S. Coast Guard, and LT Erik Oller of the U.S. Navy. The work could not have been done without the support the Office of Naval Research under Grant numbers N00014-98-1-0135 and N0001405-1-0031 whose ONR Program Manager was Dr. Thomas Swean. In addition the collegial help from Christopher Von Alt and his colleagues at the Woods Hole Oceanographic Institution is much appreciated.

\section{References}

1. Beck, R.F., Cummins, W.E., Dalzel, J.F., Mandel, P., Webster, W.C. (1989) "Motions in Waves," Principles of Naval Architecture, Vol. 3, pp. 1-190

2. St. Denis, M., Pierson, W.J. (1953) "On the Motion of Ships in Confused Seas," Trans. SNAME, Vol. 61

3. Milgram, J.H. (1995) "Extreme Tensions in Open Ocean Towing," Journal of Ship Research, Vol. 39, No. 4, pp. 328-346

4. Sclavounos, P.D., Purvin, S., Ulusoy, T., Kim, S.(2003) "Simulation Based Resistance and Seakeeping Performance of High-Speed Monohull and Multihull Vessels Equipped with Motion Control Lifting Appendages," Keynote Lecture, FAST 2003 Conference, Ichia Italy

5. Ogilvie, T.F., Tuck, E.O. (1969) “A Rational Strip-Theory of Ship Motion: Part I," Department of Naval Architecture, The University of Michigan, Report No. 013

6. Newman, J.N. (1977) "Marine Hydrodynamics," MIT Press, Cambridge, Massachusetts and London, England

7. Salvesen, N., Tuck, E.O., Faltinsen, O. (1970) "Ship Motions and Sea Loads," Trans. SNAME, Vol.78

8. von Alt, C., Allen, B., Austin, T., Forrester, N., Goldsborough, R., Purcell, M., Stokey, R. (2001) "Hunting for Mines with REMUS: A High Performance, Affordable, Free Swimming Underwater Robot," Proc. Oceans, Honolulu, Hawaii, pp. 117-122

9. Sabra, G. (2003) "Wave Effects on Underwater Vehicles in Shallow Water," Master of Science in Ocean Engineering and Master of Science in Mechanical Engineering thesis, Massachusetts Institute of Technology, Department of Ocean Engineering

10. Rybka, J.A. (2005) "A Strip Theory Approximation for Wave Forces on Submerged Vehicles in Finite Depth Water," Master of Science in Naval Architecture and Marine Engineering and Master of Science in Mechanical Engineering thesis, Massachusetts Institute of Technology, Department of Ocean Engineering

11. Oller, E.D. (2003) "Forces and Moments Due to Unsteady Motion of an Underwater Vehicle," Naval Engineer in Ocean Engineering and Master of Science in Mechanical Engineering thesis, Massachusetts Institute of Technology, Department of Ocean Engineering

12. Hoerner, S.F., Borst, H.V. (1985) "Fluid-Dynamic Lift," Hoerner Fluid Dynamics, Bakersfield, CA

13. WAMIT Version 5.4 (1998) “A Radiation-Diffraction Panel Program for Wave-Body Interactions," Massachusetts Institute of Technology, Department of Ocean Engineering 